\title{
DEUX MÉTHODES PHONOLOGIQUES POUR UNE MÊME LANGUE*
}

\section{Xiaoliang Luo}

Laboratoire Ligérien de Linguistique

\section{Résumé}

La Chine a sa propre tradition de l'étude du son, appelée yīnyùnxué : elle a pour objectif premier de contribuer à l'étude des classiques confucianistes. $\mathrm{Si}$, au début du $\mathrm{XX}^{\mathrm{e}}$ siècle, le comparatisme avait reçu un accueil favorable, le structuralisme a rencontré peu d'écho et des décennies de fermeture du pays n'ont pas permis de remédier à cette situation. Cet article analyse le mode de réception de ces théories phonologiques en Chine.

\section{Mots-clés}

Chinois, tradition, phonologie, méthodologie, post-générativisme

\section{Abstract}

China has its own tradition of the study of sound, called yinnyùnxué, its primary objective is to serve as a tool for the studies of Confucianist classics. In the early twentieth century, the comparative grammar has renewed the methodology of this discipline, however, the structuralism, marking the birth of linguistics science, has had a minor response. This article aims to understand how this separation is formed and why receptions are so different of phonological theories in China.

\section{Keywords}

Chinese, tradition, phonology, méthodologie, post-generativism

\section{INTRODUCTION}

L'étude du son des langues chinoises semble partagée en deux : la discipline héritière de la philologie traditionnelle, appelée yinnyùnxué 音韻學, s'intéresse spécifiquement, sinon exclusivement, à la diachronie ; la phonologie au sens moderne depuis le structuralisme s'intéresse à la synchronie ${ }^{1}$.

* Merci à Gabriel Bergounioux pour ses conseils et sa relecture. Je suis le seul responsable de toute erreur éventuelle de forme ou de contenu. Sauf mention contraire, la traduction des citations chinoises est mienne.

1 Nous sommes conscient que l'approche traditionnelle et la phonologie au sens moderne ne sont pas complètement étanches, par exemple, la yīnyùnxué 音韻學 a intégré certains apports 
Le présent travail essaie de rendre compte du pourquoi et du comment les éléments historiques internes et externes ont abouti à une telle partition. Dans le $\S 1$, nous présenterons brièvement la yīnyùnxué 音韻學 ; au $\S 2$, nous récapitulerons la façon dont les linguistiques « occidentales » ont été reçues au début du $\mathrm{XX}^{\mathrm{e}}$ siècle ; dans le $\S 3$, nous résumerons ce qui est advenu durant les trente ans de rupture entre la Chine continentale et le reste du monde ; au $\S 4$, nous retracerons parallèlement les avancées récentes en yīnyùnxué 音韻學 et en phonologie théorique appliquée au chinois ; au $\$ 5$, nous essaierons de comprendre cette tension entre études diachroniques et études synchroniques, entre la méthode traditionnelle et la méthode formelle, et discuterons de la possibilité de dialogue entre les deux.

\section{APERÇU DE L'ÉTUDE DU SON DES SINOGRAMMES DANS LA TRADITION CHINOISE ${ }^{2}$}

Est appelée yīnyùnxué 音韻學 la discipline traditionnelle chinoise de l'étude du son des sinogrammes. Elle est considérée, par les spécialistes chinois eux-mêmes, comme équivalant à la « phonétique historique du chinois » ( $\mathrm{Hu} 2003$, p. 2). Sa traduction en « phonologie chinoise » est due à Bernhard Karlgren et ses Études sur la phonologie du chinois ${ }^{3}$. Dans ce travail, nous considérons comme synonymes les termes yīnyùnxué 音韻學 et «phonologie chinoise ».

Cette discipline a pour objectif premier de servir d'outil pour l'étude des classiques confucianistes, doctrine officielle de la Chine pendant deux millénaires. En ce sens, elle n'est jamais parvenue à gagner son entière indépendance par rapport à la philosophie.

Elle comporte trois branches : 1) jīnyīnxué 今音學, étude du chinois médiéval ( $\mathrm{V}^{\mathrm{e}}$ au XIII ${ }^{\mathrm{e}}$ siècle, désormais $\mathrm{CM}$ ), 2) gǔyīnxué 古音學, étude du chinois archaïque (XVII ${ }^{\mathrm{e}}$ siècle av. J.-C. au III ${ }^{\mathrm{e}}$ siècle, désormais CA) et 3) děngyùnxué 等韻學, étude articulatoire et distributionnelle des attaques, rimes et tons.

\subsection{Méthode de fănqiè 反切}

Le chinois est une langue monosyllabique. L'écriture idéographique ne figure

du structuralisme et de la méthode sociolinguistique. Pourtant, la propension à opposer la tradition chinoise et les méthodes occidentales nous paraît dominante chez les chercheurs chinois en yīnyùnxué 音韻學, surtout à l'égard des courants phonologiques contemporains. Pour cette raison, nous abordons dans ce travail plutôt l'opposition que l'intégration dans la réception des théories phonologiques.

2 Nous avons consulté He (1995ab), Wang (1981), Zhao (2000) pour les éléments historiques de cet article.

3 Cette traduction n'en exclut pas d'autres. Par exemple « phonétique / phonologie historique du chinois ». Si le mot «phonologie » traduit le terme yinnyùnxué 音韻學, son emploi ici est purement conventionnel et ne restitue pas son sens moderne, celui qu'il a pris depuis le structuralisme. 
pas la prononciation mais le sens ${ }^{4}$. Afin de transcrire la prononciation d'un sinogramme, sous l'influence de la phonétique indienne en lien avec l'expansion du bouddhisme, les lettrés chinois ont inventé la méthode du fănqiè 反切 pour réaliser un sinogramme (peu courant) au moyen de deux autres (plus familiers), dont l'un partage la même consonne, l'autre la même rime, d'un monosyllabe.

Voici un exemple tiré de Tang (2002, p. 22) :

\section{東, 德紅切。}

La prononciation de 東(dōng) est donné par 德(dé), qui a la même attaque « $d$ » et 红(hóng), ayant la même rime « ong».

\subsection{Jīnyīnxué 今音學, étude $d u C M$}

Une fois la syllabe décomposée en attaque et rime, sont apparus, à l'époque du $\mathrm{CM}$, des dictionnaires où, pour servir à la composition poétique, les sinogrammes se trouvent classés en fonction de leur rime. La prononciation des sinogrammes est indiquée suivant la méthode du fănqiè 反切. La jīnyīnxué 今音學 est une étude de ces dictionnaires de rimes engagée par les lettrés sous les Ming (1368-1644) et les Qing (1644-1911) pour reconstruire le système du CM. Ils présentent un défaut : leur ambition supradialectale d'unification des langues chinoises les incite à réconcilier les systèmes de dialectes différents afin de représenter LE chinois de l'Empire.

\subsection{Gǔyīnxué 古音學, étude $d u C A$}

En les confrontant aux dictionnaires de rimes, les lettrés ont découvert que les poèmes de l'époque archaïque ne rimaient pas «correctement ». Une première explication fut que la composition de la poésie n'était pas aussi stricte à l'époque de Confucius. Au XVI siècle, la conviction s'est établie que la prononciation avait changé au fil du temps. La gǔyīnxué 古音學, entreprise par les lettrés sous les Ming et les Qing, consiste à confronter la rime des poèmes de l'époque archaïque aux dictionnaires de rimes de l'époque médiévale afin d'étudier la forme de ce qu'on appelle des xíngshēngzi 形聲字 (idéo-phonogrammes), afin de reconstruire le CA. L'une des premières découvertes fut qu'il n'y avait pas de fricative labiale en CA. Une autre conclusion importante fut que les idéo-phonogrammes ayant la même clé phonique, prononcés en $\mathrm{CM}$ avec des rimes différentes, avaient la même rime en CA.

4 À l'exception des idéo-phonogrammes. $C f$. $§ 1.3$.

5 Un idéo-phonogramme est un sinogramme construit avec une clé qui indique son sens et une clé qui donne sa prononciation. Une clé est un élément graphique qui sert à composer des sinogrammes. À titre d'exemple, 淋 lín (verser) est composé de deux clés : $\vdots$ shuǐ (eau) et 林 lín (bois), dont la première donne son sens et la seconde, sa prononciation. 
Par rapport à la jīnyīnxué 今音學, l'étude du CM, la gǔyīnxué 古音學 était plus prestigieuse : le CA, qu'on supposait parlé par Confucius, était considéré comme la langue canonique, le $\mathrm{CM}$ et les états ultérieurs du mandarin n'étant que des dégradations. Cette notion de canonicité à laquelle les lettrés étaient très attachés, a influencé jusqu'à nos jours les études linguistiques en Chine.

\subsection{Děngyùnxué 等韻學, étude articulatoire et distributionnelle}

Sous l'influence de la tradition phonétique indienne, les sons du chinois ont été décrits articulatoirement à partir des Song (960-1279). Inspirés par le tableau des syllabes du sanskrit, les děngyùntú 等韻圖 (tables de rimes) classent les sinogrammes en fonction du lieu et du mode d'articulation. Chaque rime est combinée avec toutes les attaques disponibles. Comme un sinogramme est transcrit avec deux autres suivant la méthode du fănqiè 反切, la prononciation exacte est difficile à rétablir. Les lettrés se sont donc concentrés sur une analyse phonotactique : certaines attaques ne se combinent jamais avec certaines rimes ; certaines attaques se combinent avec des rimes avec lesquelles d'autres attaques ne peuvent se combiner. Il y a une reconnaissance de la complémentarité distributionnelle.

\section{RENCONTRE DE DEUX MONDES}

À partir du milieu du XIX ${ }^{\mathrm{e}}$ siècle, en Chine, l'incursion de l'Occident s'est accompagnée de la diffusion de la modernité : d'abord les techniques et l'industrie, ensuite les conceptions marchandes modernes et enfin les valeurs et les savoirs. Il faut attendre les années 1910, après la chute de l'Empire mandchou (1644-1911) et la fondation de la République de Chine (1912-1949), pour que la philosophie, la logique, les sciences naturelles et les sciences humaines soient introduites de façon systématique en Chine, y compris la linguistique.

\subsection{Rencontre de deux phonologies : années 1919-1949}

Les relations entre la phonologie (au sens large) occidentale et la yinnyùnxué 音韵學 sont unidirectionnelles : à part les sinologues, les savoirs issus de la tradition chinoise sont peu connus en Occident, alors que la yīnyùnxué 音韵學 a été très influencée par la linguistique moderne.

Dans la première moitié du $\mathrm{XX}^{\mathrm{e}}$ siècle, la linguistique comparée est stabilisée. Au niveau méthodologique, elle reconduit les procédures éprouvées sur les langues indo-européennes en comparant toujours plus de langues et en étudiant leur généalogie afin de reconstruire des super-familles. En 1929, le centre de recherche le plus important pour la yīnyùnxué 音韻學, l'Institute of History and Philology 
(Lish ̌ yŭyán yanjiūsuŏ) de l'Academia Sinica, est fondé. Linguistiques comparée et structurale et phonétique instrumentale sont confrontées à la yīnyùnxué 音韻學 et contribuent à son actualisation, mais de façon très différenciée. Les phonologues chinois ainsi que les historiens de la linguistique parlent souvent de « linguistique moderne » sans opérer de distinction entre le comparatisme et le structuralisme ni spécifier leur influence respective sur l'étude du chinois ${ }^{6}$.

Nous essaierons de rétablir les distinctions qui s'imposent.

\subsection{Influence de la phonétique}

Les différentes branches de la phonétique ont infléchi la tradition chinoise :

- la phonétique articulatoire en renouvelant la terminologie ;

- la phonétique acoustique en rendant compte des phénomènes tonals ;

- l'introduction de l'API en permettant la transcription fiable des langues chinoises.

Les termes utilisés pour décrire la phonation dans děngyùnxué 等韻學 sont souvent polysémiques et varient d'un auteur à l'autre. La phonétique articulatoire a permis une clarification de la terminologie. Désormais on pourra interpréter les termes en se basant sur les acquis de la phonétique historique du chinois : par exemple, le même terme zhuó 濁 (troubles) qui désignait des séries différentes de consonnes d'une époque à une autre, sera restitué comme " voisé » (VII ${ }^{\mathrm{e}}$ siècle) ou « soufflé » (VIII ${ }^{\mathrm{e}} \mathrm{XI}^{\mathrm{e}}$ siècles) (cf. Ferlus 2009, p. 194-195).

On peut faire remonter la prise de conscience des tons au $V^{\mathrm{e}}$ siècle mais personne n'avait entrepris de les caractériser scientifiquement. La description des quatre tons du CM : plat, montant, partant et rentrant, était impressive et métaphorique. Voici comment il en était rendu compte :

平聲哀而安，上聲厲而舉，去聲清而遠，入聲直而促。(處忠《元和韻譜》)

Le ton plat est pathétique et paisible, le ton montant féroce et soulevant, le ton partant clair et lointain, le ton rentrant direct et court. (Chu Zhong, Yuánhé Yùnpŭ )

ou encore

平聲長而空，如擊鐘鼓，上去入短而實，如擊木石。(江永《音學辨微》)

Le ton plat long et vide comme le tambour, les tons montant, partant et rentrant sont courts et solides, comme frapper le bois et la pierre. (Jiang Yong, Yīnxué Biànwēi )

6 Cette confusion continue. Zhu (2013) pense que le comparatiste Karlgren ( $c f$. infra) serait influencé par Saussure pour reconstruire le chinois ancien et que la loi de Grimm serait du structuralisme. Or, le premier volume de ses Études sur la phonologie chinoise a été publié un an avant les Cours de linguistique générale. En 1940, Chao Yuen-Ren, a proposé de réduire la reconstruction phonétique de Karlgren, purement comparatiste, en reconstruction phonémique, sous l'influence de la version bloomfieldienne du structuralisme. $C f$. Malmqvist (2011, p. 174). 
Ici le timbre (clair, tambour, bois, pierre), la longueur (long, court) et l'intensité (loin) se trouvent confondus avec la hauteur (paisible, soulevant).

En 1912, le phonéticien anglais Daniel Jones a publié avec Kwing Tong Woo $A$ Cantonese phonetics reader, un manuel de conversation en cantonais pour débutants, dans lequel ils schématisent le contour tonal du cantonais par la portée musicale (Jones \& Woo 1912), spécifiant sa signature fréquentielle. Ce livre a eu peu d'écho en Chine (aucune mention dans les histoires de la linguistique chinoise que nous avons consultées). Les raisons qu'on peut avancer sont les suivantes : premièrement, à l'Academia Sinica, la spéculation était prestigieuse, l'application et la vulgarisation beaucoup moins ( $c f$. Zhao 2000, chap. 6.1). Un manuel de langue n'était pas considéré comme un travail de recherche. Deuxièmement, le travail en diachronie était valorisé alors que les études synchroniques étaient mal reçues. Troisièmement, pour les linguistes chinois de cette époque, les études dialectales n'avaient d'intérêt qu'en lien avec la reconstruction du chinois ancien.

C'est dans les années 20 que les tons ont été décrits adéquatement. Liu Fu en France et Chao Yuen-Ren en Allemagne ont étudié la phonétique de laboratoire et ils ont procédé à des analyses expérimentales. Leurs analyses montrent que les tons sont corrélés à une variation de hauteur dessinant des contours distinctifs. Il n'y a pas de preuve que leur travail se soit inspiré de celui de Daniel Jones mais la quantification des contours tonals du mandarin pékinois (appelé aujourd'hui « chinois standard ») et potentiellement celui de tous les systèmes tonals dialectaux était établie.

La méthode fănqiè 反切 supposait que le locuteur connaisse préalablement la prononciation des sinogrammes donnés en référence. Cette méthode ne vaut pas pour le locuteur d'un autre système dialectal qui ne reconnaîtrait pas la prononciation des deux sinogrammes transcripteurs. Les symboles de l'API ont permis de proposer une restitution de la valeur phonétique indépendante de toute lecture dialectale. Ils se sont très vite répandus chez les phonologues en raison de leur commodité d'emploi pratique et de leur économie même si les lettrés formés dans la phonologie traditionnelle y résistaient ${ }^{7}$.

\subsection{Karlgren et les méthodes comparatistes}

Si la phonétique a pu actualiser le métalangage et la technique de transcription de yīnyùnxué 音韻學, le comparatisme a renouvelé sa méthodologie. À cette époque plusieurs sinologues européens ayant reçu une formation comparatiste ont travaillé sur la phonologie chinoise. Le plus important d'entre eux est Bernhard Karlgren. He (1995b, p. 231) considère même que les années 1920 à 1949 sont celles de l'ère de Karlgren dans l'histoire de la phonologie chinoise.

$\mathrm{Au}$ début $\mathrm{du} \mathrm{XX}^{\mathrm{e}}$ siècle, la méthode comparative n'avait pas encore été

7 Les phonéticiens et phonologues qui ont adopté l'API ont été appelés, avec mépris, des APIiste, par les lettrés qui défendent la tradition (Zhao 2000, p. 486). 
appliquée aux langues chinoises. Ayant étudié le russe et la phonologie historique en Suède, Karlgren a résolu d'appliquer les méthodes comparatistes au chinois ${ }^{8}$. À ce titre, il a été le premier à étudier les dialectes vivants pour reconstruire le CM quand les lettrés chinois étudiaient prioritairement, sinon exclusivement, la langue écrite, sans attention pour les dialectes, car leur objectif était de restituer la langue parlée par Confucius, le seul état de langue valorisé 9 .

La grammaire comparée a permis de montrer que la comparaison des langues vivantes apparentées facilitait la reconstruction d'un état précédent commun conjecturé (proto-langue). Ainsi a-t-il mené lui-même des enquêtes sur une dizaine de dialectes du nord et collecté de seconde main les données de plusieurs dialectes du sud, pour les comparer et décrire ce qu'il suppose être leur provenance commune : le CM.

Karlgren est aussi le premier à utiliser les emprunts chinois dans les langues voisines (cf. Zhao 2000, chap. 6). L'ethnocentrisme des lettrés chinois avait pour résultat une indifférence certaine à l'égard des langues environnantes, alors que les emprunts conservent souvent la trace d'une forme plus ancienne. Karlgren a étudié systématiquement les emprunts chinois en japonais, coréen et vietnamien pour les comparer avec les données dialectales. Il a confronté ses données transcrites en API avec le dictionnaire de rimes Guăngyùn 廣韻 et quelques tables de rimes, et il a pu proposer une reconstruction du $\mathrm{CM}$ avec, pour la première fois, une indication de la valeur phonétique de chacun des sinogrammes. Il est également le premier à donner une explication des sìděng 四等 (quatre divisions), une description articulatoire dans les tables de rimes. Celles-ci n'avaient jamais explicité selon quel(s) critère(s) était opéré le classement. Karlgren a essayé de démontrer que plusieurs critères avaient été confondus : la différence de la «médiane » (le glide avant la voyelle) et le timbre du noyau (voyelle) ${ }^{10}$.

La valeur phonétique des unités qu'il est amené à proposer dans sa reconstruction des quatre divisions est très ad hoc, souvent imprononçable (par exemple la succession de jod et de w devant une autre voyelle), mais le point de vue selon lequel on doit dissocier deux critères confondus a été largement accepté ${ }^{11}$.

8 Selon Malmqvist (2011, chap. 1), la raison pour laquelle Karlgren décida de s'engager dans l'étude du chinois est que son professeur Lundell, comparatiste russe enseignant en Suède, lui dit que les dialectes chinois avaient un besoin immanent d'enquête.

9 Sur la canonicité de la langue parlée à l'époque de Confucius, $c f$. une analyse détaillée par Martin (1988 et 1990).

10 Voici un exemple de quatre divisions tiré et adapté de Tang (2002, p. 69) :

\begin{tabular}{|c|c|c|c|c|}
\hline & Division I & Division II & Division III & Division IV \\
\hline $\mathrm{CM}$ reconstruit & 高[kau] & 交[kau] & 嬌 $[\mathrm{ki \varepsilon u}]$ & 堅[kieu] \\
\hline Mandarin & [kao] & [tcao $]$ & [t6ao] & [tढen] \\
\hline
\end{tabular}

11 Une avancée récente sur la reconstruction des quatre divisions est proposée Ferlus (2009) qui se fonde sur le modèle développé à partir des langues mon-khmer. 
Karlgren a aussi, de façon plus générale, contribué à la reconstruction du CA à partir du système de $\mathrm{CM}$ conjecturé.

Pendant une trentaine d'années, les phonologues chinois ont étudié l'approche comparatiste de Karlgren qu'ils ont discutée, modifiée et traduite. Son influence peut s'expliquer par la reconfiguration qu'ont produite des données nouvelles (le chinois) sur une école linguistique façonnée par d'autres langues (la linguistique indo-européenne). Il n'a pas en retour fait progresser la théorie linguistique dont il s'inspirait puisqu'il s'agit d'une application unidirectionnelle. Si sa reconstruction des segments était exhaustive, au niveau tonal sa réflexion est entravée par le modèle à partir de quoi s'est développée la linguistique historique.

Comment expliquer que Karlgren ait été accueilli favorablement par les linguistes chinois ? Il semble que ce soit parce qu'il a apporté ce dont ils avaient le plus besoin. La reconstruction est au cœur de yīnyùnxué 音韻學. Karlgren a étudié systématiquement les travaux des lettrés chinois ; il adoptait leur terminologie et utilisait les sinogrammes en suivant leur arrangement dans les dictionnaires de rimes. Le travail diachronique, comme nous l'avons mentionné, jouit traditionnellement d'un prestige dû à l'idée de canonicité très prégnante chez les lettrés chinois. La dialectologie a gagné en légitimité après les travaux de Karlgren, au moins partiellement, parce que désormais il y a une prise de conscience de son importance pour le travail de reconstruction. Nous comprendrons mieux la situation historique du succès de Karlgren si nous le rapprochons de la réception de Charles Hockett.

\subsection{Une nouvelle méthode : le structuralisme}

En linguistique, les trente premières années du siècle dernier sont marquées par le déclin du comparatisme et la montée du structuralisme. Trois écoles structuralistes se sont développées indépendamment en Europe de l'Ouest (Saussure et ses élèves), Europe de l'Est (le Cercle Linguistique de Prague) et aux États-Unis (Boas, Sapir).

À cette époque, la Chine connaît une transformation de son système d'éducation. Les écoles traditionnelles sont remplacées par des universités qui reprennent le modèle occidental. Les linguistes de cette époque ont été en général formés d'abord par les écoles traditionnelles, puis par les universités. Ce type de cursus permet de mieux comprendre la transmission du structuralisme en Chine. Deux grands linguistes ont été formés aux États-Unis, Chao Yuen-Ren et Li Fang-Kuei, qui ont transmis les premiers les principes structuralistes en Chine, inspirés de la linguistique descriptive de corpus des États-Unis.

Li Fang-Kuei a étudié auprès de Sapir entre 1924 et 1928 aux États-Unis. Il est l'auteur de plusieurs travaux importants de description des langues amérindiennes. Après son retour en Chine en 1929, il a eu recours à une méthode descriptive pour 
étudier les langues tai-kadai ${ }^{12}$ et les langues sino-tibétaines.

Chao Yuen-Ren a étudié la linguistique à Harvard. Ses travaux concernent d'abord la phonétique expérimentale dans les années 1920, au cours de son séjour aux États-Unis et en Europe. Il s'intéresse en particulier à la quantification des niveaux tonals. Il a connu les idées structuralistes à partir des années 30 en prenant connaissance du Language de Bloomfield puis du cercle linguistique de l'Université de Yale (dont faisait partie Bloomfield) et il a en appliqué les principes en dialectologie. Il est un des premiers à introduire le concept de « phonème » et la méthode descriptive en Chine ( $c f$. Chao 2002). Chao Yuen-Ren accorde une importance toute particulière à la phonologie historique :

nous devons nous demander quels sont les initiales, les rimes, lestons et leur combinaison, quel sinogramme appartient à quelle catégorie de rimes, la différence de ces catégories de rimes avec d'autres dialectes, et avec celles de l'ancien chinois. (Chao 2002, p. 273)

Ceci est encore plus net chez Luo Changpei. Formé à l'Université de Pékin à la fin des années 1910, où il a étudié la phonologie chinoise et la linguistique occidentale, il a contribué à la reconstruction de l'ancien chinois, à la description des dialectes et des langues minoritaires ${ }^{13}$. Son objectif premier est la reconstruction de l'ancien chinois. Par exemple, Luo (1956) compare systématiquement toutes les initiales et rimes du dialecte de Xiamen (province du Fujian) avec ceux du dictionnaire de rimes Guăngyùn 廣韻. À la lecture des histoires de la linguistique chinoise, on relève que seul Wang (1981) opère une distinction tranchée entre la linguistique comparée et la linguistique descriptive et qu'il est conscient de la contribution de chacune des méthodes. L'auteur en a fait ce commentaire à propos de la description des dialectes :

Les enquêtes dialectales avant la Libération ont deux défauts : (..) Deuxièmement, ce n'était pas de la linguistique descriptive pure, la linguistique historique interfère. (...) Le but de nos enquêtes dialectales n'est pas d'étudier l'ancien chinois (du moins ce n'est pas le but principal), il n'est donc pas nécessaire de comparer les dialectes avec l'ancien chinois (...) [certains travaux sur les dialectes] n'ont pas de description synchronique ; (...) En effet, le travail sur les dialectes n'a pas besoin de Guăngyùn 廣韻, (...) (Wang 1981, p. 203)

L'influence du structuralisme, dans sa version américaine, est moins importante que celle de la linguistique comparée. Cette influence est circonscrite à la description des dialectes, conformément à la tradition chinoise. La méthode descriptive est surtout synchronique et fondée sur des témoignages oraux. Elle n'attire l'intérêt des linguistes chinois que lorsqu'elle peut contribuer à éclairer des processus diachroniques.

12 Langues parlées essentiellement en Asie du Sud-est et en Chine du sud, dont le regroupement généalogique reste l'objet de discussions.

13 Langues parlées par des peuples minoritaires en Chine, i.e. autres que les Hàn 漢, ethnie historiquement chinoise. Luo Changpei a étudié, pendant son séjour dans la province du Yunnan, au sud-ouest de Chine, notamment le yi et le drung (langues tibéto-birmanes). 


\subsection{Un nouveau domaine : l'étude du mandarin pékinois}

Les dialectes du nord étaient depuis longtemps considérés comme des langues « contaminées » par l'accent des peuples nomades, les études sur ces dialectes méprisés étaient négligées. Depuis les années 1920, l'État chinois a essayé de généraliser le pékinois en tant que langue nationale, mais sa fonction a toujours été considérée comme véhiculaire et il ne jouissait d'aucun prestige particulier. Pour cette raison, lorsque la plupart des linguistes chinois s'engageaient dans l'étude diachronique de l'ancien chinois, l'étude du pékinois suscitait peu d'intérêt. Pourtant, les travaux qui y sont consacrés pendant cette période marquent le début d'un nouveau domaine. La version standardisée du pékinois deviendra ce qu'on appelle aujourd'hui le « chinois standard».

Des années 20 jusqu'au début des années 50 , les études sur le pékinois sont de deux types : l'étude comparatiste et l'étude descriptive. L'étude comparatiste consiste à décrire le système du pékinois dans le cadre diachronique et dialectologique pour en retrouver l'origine et comprendre les changements phonétiques qu'il a subis, ou encore justifier son statut. Par exemple, en 1937, Wei Jiangong a tracé les grandes lignes de la genèse du pékinois et a conjecture à son origine un mélange de dialectes chinois, une koinê sur laquelle les langues des peuples nomades avaient eu peu d'influence (He 1995b, p. 380-381). Un pékinois sans mélange de « barbare », purement chinois, a toute légitimité pour être érigé en langue officielle et jouir d'un prestige mérité.

L'étude descriptive est au contraire synchronique. À cette époque, presque aucun linguiste chinois ne s'y intéressait. Le seul travail qu'on pourrait mentionner est celui de Chao (1929) qui décrit le système de tons et d'intonation du pékinois mais qui adjoint une partie diachronique pour trouver la correspondance des tons pékinois dans le système médiéval. Il faut attendre son installation définitive aux États-Unis pour que Chao écrive des travaux de référence sur la synchronie du mandarin pékinois.

Les linguistes descriptivistes américains présents en Chine dans les années 40 - Hartman (1944), Hockett (1947 et 1950) - n'ont en revanche aucune intention de corréler la description synchronique à la tradition chinoise en diachronie. Hartman (1944) étudie la phonologie segmentale du dialecte pékinois et dresse une liste de tous les sons ; il analyse leur distribution et combinaison pour caractériser tous les phonèmes. Hockett (1947) offre une description plus exhaustive du pékinois dans tous ses aspects : segments, intonation, tons. Il analyse également la distribution et la combinaison de ces éléments en introduisant la notion de trait distinctif puis confronte son analyse à celle de Hartman (1944). Hockett (1950) poursuit dans cette voie mais en se centrant sur la morphophonologie. Il se propose d'appliquer la méthode développée par Zellig S. Harris dans l'analyse de la morphologie en se basant sur les résultats de Hockett (ibid.) pour la phonologie, afin d'analyser les alternances phonologiques dans les processus morphologiques. Comme il n'y 
avait guère de place pour la morphologie dans la linguistique chinoise de ce temps, ce travail est pionnier.

Les articles de Hartman et de Hockett sont les premières vraies études structuralistes qui dépassent résolument le cadre comparatiste. Ces travaux n'ont eu presque aucune répercussion auprès des linguistes chinois, d'autant que la situation politique allait transformer les conditions des échanges scientifiques.

\subsection{Le début de deux phonologies}

Aucune version de l'histoire de la linguistique chinoise ne mentionne les travaux de Hartman et de Hockett. Même He (1995b), qui consacre une section aux études sur le pékinois, n'y fait pas allusion alors qu'il fait référence à Karlgren. Toutes les études sur l'histoire de la linguistique chinoise parlent de la modernisation de la phonologie chinoise par les apports de la linguistique moderne, sans préciser de quelle linguistique moderne il s'agit. Autrement dit, avec quelle linguistique moderne yinnyùnxué 音䫓學 serait-elle compatible ? Le comparatisme partage avec la tradition chinoise un même objectif de reconstruction. Le structuralisme s'intéresse en priorité à la synchronie. Il y a donc eu deux réceptions très différentes du comparatisme et du structuralisme : la phonologie chinoise, centrée sur la diachronie, représentée par Karlgren, et la phonologie du chinois, centrée sur la synchronie, représentée par Hockett.

Longtemps, la phonologie chinoise a été la seule phonologie du chinois. Le mot phonologie a été traduit par yinnyùnxué 音韻學. À partir du moment où la phonologie chinoise s'est avérée rétive au structuralisme, le terme phonologie du chinois a gagné sa légitimité et son indépendance en désignant spécifiquement cette phonologie du chinois que la phonologie chinoise rejette avec un certain mépris, même si le terme approprié pour la traduire, yinnxìxué 音系學, ne se stabilisera que dans les années 1980.

Il est vrai que Chao Yuen-Ren et Li Fang-Kuei prouvaient, par leurs travaux, que ces deux phonologies peuvent coexister chez un même chercheur, mais malheureusement ils sont restés isolés dans leur double compétence en phonologie chinoise et en linguistique structurale. Le manque de dialogue entre les deux phonologies sera aggravé par trente ans d'isolement de la Chine Populaire. 


\section{RUPTURE : DEUX MONDES QUI S'IGNORENT}

\subsection{Sur le continent}

Dans certaines histoires de la linguistique chinoise, la description de cette période est paradoxale : dans les paragraphes d'introduction ou de résumé, on trouve des déclarations telles que "Après la Libération, sous la direction du Parti, la linguistique chinoise a connu un développement sans précédent. » (Wang 1981, p. 213). Mais les détails qu'exposent ces auteurs semblent montrer le contraire : « Dans le climat [politique] global, ils [les linguistes] n'ont pas pu utiliser leur talent » ou « pendant la Révolution culturelle, la recherche en linguistique a été interrompue » (Zhao 2000, p. 480).

Ces trente ans constituent une période où la recherche scientifique s'est trouvée isolée du reste du monde ; il n'y avait presque aucune interaction avec le milieu linguistique occidental (Tang \& Geng 1998).

Durant les quelques années qui ont suivi la Libération, la Chine s'est tournée vers l'URSS. La remise en cause, d'inspiration politique, concernait le champ de la linguistique. La science était considérée comme ayant un caractère de classe. On opposait la science féodale, la science bourgeoise et la science prolétarienne. Quant à la linguistique, non seulement la discipline, mais la langue elle-même était considérée comme ayant un caractère de classe. On distinguait donc une linguistique féodale (les études traditionnelles), une linguistique bourgeoise (la linguistique générale) et une linguistique socialiste, inspirée par le marxisme au moins dans sa version stalinienne. Tous les chercheurs qui, issus de la République de Chine, ont contribué à la science bourgeoise, ont dû « abjurer» la science bourgeoise. De 1950 à 1958, avant la rupture de la Chine avec l'URSS, la linguistique socialiste en Chine a suivi les fluctuations des débats en URSS autour du marrisme ${ }^{14}$, par exemple les discussions concernant le caractère de classe de la langue.

En 1956, Mao a approuvé le système de pinyin actuellement en vigueur, « notre propre » romanisation du chinois contre les romanisations des « impérialistes », et une première version a été appliquée. En 1958, cette version du pinyin a été imposée comme romanisation officielle du mandarin et enseignée dans toutes les écoles du pays.

Soutenu par l'État, le nombre d'enquêtes dialectales réalisées a été considérable. Pour la première fois, une cartographie détaillée des dialectes et des langues minoritaires a été dessinée et il y a eu une profusion de travaux sur la généalogie des dialectes.

En phonologie, à part quelques rééditions, peu d'ouvrages importants ont paru à cette époque. La recherche devait se conformer à la politique, par exemple, dans

14 La doctrine officielle en URSS à cette époque était celle de Nicolas Marr (1864-1934), qui considère qu'il y a une nature de classe des stades du langage. 
Wang (1980 [1957]), une étude approfondie sur l'évolution du CA vers le chinois moderne, dans laquelle l'auteur propose de nombreux points de vue originaux sur la reconstruction, les premières pages foisonnent de citations de Staline et de Lénine.

$\mathrm{Si}$, avant 1966, les chercheurs pouvaient poursuivre leurs activités pour peu que leur travail soit «politiquement correct », les dix ans de la Révolution Culturelle (1966-1976) sont marqués, dans le domaine de la linguistique comme dans tout le champ du savoir, par une course au néant. Pendant ce temps, en Occident, la phonologie structuraliste cédait la place à la phonologie générative, une réalité totalement ignorée par les chercheurs chinois et il faut attendre la fin de la Révolution Culturelle pour que la Chine découvre que le reste du monde a changé.

\section{2. À l'extérieur}

Pendant ces trente ans, la recherche hors du territoire continental s'avérait productive. Du côté de l'étude diachronique, non seulement la reconstruction du $\mathrm{CM}$ a pu être réalisée mais celle du CA a connu de réelles avancées ${ }^{15}$.

Du côté de l'étude synchronique, la méthode structuraliste des travaux de Hartman et de Hockett a servi de modèle. Chao (1968, chap. 1), après une description phonétique de l'inventaire des sons, des tons, accent et intonation, analyse leur distribution et leur combinaison, en s'affranchissant de la diachronie et du comparatisme. Aux États-Unis, la linguistique descriptive a établi et perfectionné une méthodologie qui avait été appliquée à un certain nombre de langues. Les débats portent sur des détails tels que le décompte des phonèmes.

La phonologie générative a influencé l'étude du chinois en dehors du territoire chinois dans les années 60-70. On peut citer Woo (1969) sur le changement tonal de chaîne, Cheng (1973) sur les diphtongues et le ton neutre, Dell (1973) sur le sandhi tonal des formes dupliquées en mandarin. Ces travaux ne décrivent pas les unités linguistiques en tant que telles mais exploitent les aspects dynamiques du chinois, l'interaction entre ces unités dans les concaténations.

Néanmoins, la phonologie générative n'a pas eu beaucoup d'écho auprès des linguistes chinois. D'abord, la structure de l'anglais est bien connue et $S P E^{16}$ se propose d'expliquer les règles de l'accentuation de l'anglais, ce qui n'a guère de raison de susciter l'intérêt des linguistes chinois. En diachronie, ils ont pour souci de décrire le système médiéval ou archaïque ; en synchronie, le mandarin pékinois n'a gagné sa légitimité en tant qu'objet d'étude que depuis peu, aussi la tâche la plus urgente était de décrire sa structure adéquatement, et non pas d'expliquer un phénomène particulier. Ainsi, la phonologie générative ne répond à une attente

15 Nous regroupons les études diachroniques de l'Après-guerre au $\S 4.1$.

16 Chomsky, N et Halle, M (1968). The Sound Pattern of English. New York: Harper \& Row. Euvre fondatrice de la phonologie générative. 
ni diachronique ni synchronique des linguistes chinois. Ensuite, la phonologie générative est unilinéaire et explique souvent de façon ad hoc les phénomènes tonals. Enfin, la Chine à cette époque est isolationniste et la phonologie générative est ignorée des linguistes du continent.

\section{DEUX PHONOLOGIES QUI SE DÉVELOPPENT INDÉPENDAMMENT}

\subsection{Les avancées récentes en yīnyùnxué 音韻學}

Héritière de la tradition lettrée, intégrant la méthode comparatiste et certains apports de la phonologie structurale, yinnyùnxué 音韻學 a réalisé certaines avancées sur plusieurs points.

La reconstruction du $\mathrm{CM}$ a abouti à un système complet, nous pouvons citer entre autres les travaux de Edwin G. Pulleyblank, considérés comme des références.

Celle du CA a suscité un grand intérêt chez les linguistes et a connu un fort développement depuis 1945. André-Georges Haudricourt, inspirés par les études sur les langues mon-khmer, conjecture pour la première fois une tonogénèse du $\mathrm{CA}$ vers le $\mathrm{CM}$, consécutive à la chute de la consonne finale.

Depuis Karlgren, il a été conjecturé que le CA avait des groupes consonantiques et une morphologie plus riche. Ce point de vue est largement, mais non sans réserves, partagé par de nombreux chercheurs. Nous pouvons citer entre autres les travaux de Laurent Sagart (1999) sur la morphologie et la phonologie du CA.

L'étude de l'aire linguistique d'Asie orientale et du sud-est continue à contribuer de façon significative à la reconstruction de l'ancien chinois. Si les quatre divisions du CM n'ont pu être déterminées, c'est que les linguistes sont le plus souvent partis du CM ; Ferlus (2009), sur le modèle développé à partir des langues monkhmer, propose une solution des quatre divisions en prenant l'itinéraire inverse, du CA au CM, dans le passage du sesquisyllabisme vers un strict monosyllabisme.

La reconstruction du CA est considérée dans le contexte plus large sinotibétain. Les avancées en CA bénéficient des études sino-tibétaines et vice versa (cf. Gong Hwang-cherng 2004).

\subsection{Les nouvelles phonologies et le chinois}

Nous appelons «nouvelles phonologies » les courants post-génératifs développés à partir de la phonologie auto-segmentale. Les études du chinois dans le cadre des nouvelles phonologies sont essentiellement synchroniques ou dialectales.

L'année qui marque le terme de la Révolution Culturelle est celle durant laquelle John Goldsmith a soutenu un doctorat qui fonde la phonologie autosegmentale. En reprenant ce cadre, Yip (1980) a soutenu la première thèse sur le chinois dans le cadre post-génératif qui traite les phénomènes tonals du mandarin et du cantonais dans un cadre multilinéaire. Au cours des années 1980, la Chine a envoyé des 
étudiants dans les universités occidentales. Quelques années après paraîtront au MIT les recherches de Duanmu (1990) et Bao (1990).

Après trente ans d'interruption des échanges, la linguistique formelle paraissait peu accessible pour les linguistes chinois et n'intéressait que peu de chercheurs. Wang Jialing, un professeur d'anglais, a longtemps été seul à représenter les courants phonologiques modernes. À partir de 1993, quand la Théorie de l'Optimalité (OT) s'est imposée en phonologie, c'est encore Wang qui l'a rendue familière au public chinois. Son élève, Ma Qiuwu, angliciste, a pris le relais et a présenté OT de façon systématique dans les premières années du siècle en l'appliquant aux dialectes chinois. Avant lui, Wang Hongjun (Wang 1999), professeur au Département de chinois de l'Université de Pékin, avait étudié au MIT en tant que chercheur invité, et elle a présenté plusieurs des courants phonologique qui avaient précédé OT.

Dans le même temps, aux États-Unis, plusieurs thèses ont été soutenues sur la phonologie du chinois, le plus souvent dans le cadre d'OT, et de nouvelles recherches ont fait l'objet d'une publication : Duanmu (2007 [2000]), Bao (1999), Yip (2002).

En France, parmi les travaux dans le cadre post-génératif, on peut citer Dell (1994; 2004). En 2008, une thèse, Liu (2008), dans le cadre du modèle CV à contours, a été soutenue à Paris 8 .

La phonologie du gouvernement (désormais PG) a été élaborée à partir de langues afro-asiatiques dont la structure est très éloignée de celle du chinois. L'élève de Jonathan Kaye, Goh Yeng-Seng, est le premier à en faire l'application au chinois dans une thèse soutenue en 1996 à la SOAS, à Londres, et publiée à Taipei (Goh 1997). En 1999, Kaye s'est installé en Chine pour enseigner la phonologie et il s'intéresse au chinois depuis cette date. Ses analyses sont exposées dans Kaye (2001ab).

Les travaux conduits dans le cadre des nouvelles phonologies se concentrent sur trois questions :

Phénomènes tonals : Les études sur les langues africaines ont beaucoup contribué à la tonologie dans le cadre multilinéaire, mais la nature ponctuelle des tons de ces langues diffère des tons modulés des langues chinoises. Dans un premier temps, les sinisants ont essayé de transposer aux tons modulés les études conduites sur les tons ponctuels. Le travail pionnier de Yip, ainsi que les travaux de Duanmu et de Bao, ont essayé de concilier la représentation phonologique avec la valeur phonétique des cinq niveaux proposée par Chao. Jusqu'à Duanmu ([2000] 2007), considéré comme une référence dans le domaine, la plupart des chercheurs ont discuté les tons chinois dans le cadre de la géométrie des traits, notamment Bao (1990), Duanmu (1990) et Yip (1995). Ces études des phénomènes tonals ont été très tôt étendues au-delà du mandarin. Les systèmes dialectaux ont fait objet de monographies, puis des études inter-dialectales sont apparues qui se sont souvent focalisées sur un seul phénomène tonal, par exemple, le sandhi tonal, commun à beaucoup de dialectes chinois (Chen 2000). Une partie importante des données ont 
été collectées par des chercheurs chinois, publiées en Chine, puis reprises par les chercheurs étrangers afin d'élaborer une analyse formelle.

Kaye (2001b), dans le cadre de la phonologie du gouvernement (PG), essaye de générer le système tonal du mandarin et du cantonais en définissant les unités primitives, communes à toutes les langues, et les paramètres, propres à une langue donnée. Liu (2008), dans le cadre du modèle CV à contours, propose l'existence d'un squelette tonal $\mathrm{HBHB}^{17}$ pour expliquer les phénomènes de sandhi tonal.

Distribution et combinabilité : Dans le cadre structuraliste, la phonologie segmentale du pékinois a déjà fait l'objet de plusieurs études de Hartman, Hockett, Chao et Cheng. Ces travaux décrivent les combinaisons consonne-voyelle pour étudier leur distribution et leur complémentarité et pour inventorier les phonèmes. Ils n'expliquent pas pourquoi certaines combinaisons sont bien formées alors que d'autres, tout à fait prononçables, sont mal formées. Dans le cadre des nouvelles phonologies, la description n'est plus le seul souci des chercheurs. Ceux-ci cherchent aussi à trouver une explication, souvent dans le cadre de la géométrie des traits et d'OT. Pour la géométrie des traits, il s'agit de caractériser les traits dont l'incompatibilité prédit quelles seront les combinaisons non recevables. Pour OT, il s'agit de définir une série de contraintes afin de sélectionner les bons candidats.

Toujours dans le cadre de la PG, pour Kaye (2001a), la combinabilité est un problème d'association et de propagation des éléments primitifs (Théorie des Éléments). Toute attaque peut se combiner avec tout noyau dans la structure sous-jacente. La compatibilité entre l'attaque et le noyau est ainsi réduite à la propagation d'un élément déjà contenu dans l'attaque vers une position vocalique.

Accent tonique et interface phono-syntaxe : Les études sur l'accent tonique et son interaction avec la syntaxe sont menées dans le cadre de la théorie métrique. On citera Duanmu (2007 [2000], chap. 6) pour l'accent tonique et les critiques de Dell (2004). L'existence de l'accent tonique du chinois standard reste un débat ouvert. Feng Shengli, élève de Liberman, travaille sur la syntaxe diachronique et synchronique du chinois d'un point de vue prosodique. On peut citer également Feng (1997).

\section{DEUX PHONOLOGIES QUI S'IGNORENT}

Les travaux dans le cadre des nouvelles phonologies sont totalement ignorés par yīnyùnxué 音韻學 d'aujourd'hui. Aucune version de l'histoire de la linguistique chinoise ou de la phonologie chinoise ne les mentionne.

Il y a à cet état de fait des raisons internes et externes. Les raisons internes tiennent pour une grande part à l'approche traditionnelle chinoise. Les raisons externes dépendent de l'organisation des institutions d'enseignement et de recherche. 
La spécialisation des départements dans les établissements d'enseignement supérieurs chinois, calqués sur le système soviétique, a conduit à affecter les linguistes dans les départements de chinois ou de langues étrangères qui relèvent d'institutions séparées. Par exemple, l'Université de Nankin, un des foyers de la linguistique chinoise depuis les années 1920, a actuellement deux laboratoires de linguistique, l'un fait partie du département de chinois, étudiant uniquement la phonologie chinoise, l'autre regroupe tous les linguistes des départements d'anglais, de français, d'allemand etc., étudiant uniquement la linguistique formelle et étrangère. Les sinisants n'ont pas de formation en linguistique formelle, les linguistes qui font de la linguistique formelle n'ont pas de formation en yīnyùnxué 音韻學. À Taïwan, l'installation de l'Academia Sinica après le repli du Kuomintang a repris le système d'avant 1949, dont l'Institute of History and Philology (Lishǐ yŭyán yanjiūsuŏ), pour traiter surtout de la méthode comparatiste. En 2004, un Institute of Linguistics a été fondé, où sont menées des recherches en linguistiques expérimentales, théoriques et cognition.

\subsection{Yīnyùnxué 音韻學 face à elle-même}

Tang (2002) est le manuel universitaire de référence de yinnyùnxué 音韻學 ; ses points de vue sont représentatifs des orientations de cette discipline. Aujourd'hui, yinnyùnxué 音韻學 se considère comme « une discipline qui étudie la prononciation des sinogrammes et leur changement historique. » (Tang 2002, p. 1). La langue reste inséparable de l'écriture.

Dans Tang (2002, p. 4), l'auteur superpose la synchronie et la diachronie :

Quand on analyse la structure phonétique du chinois moderne, ona alors affaire à la phonologie historique. Si l'on veut vérifier la combinaison des consonnes, voyelles et tons du chinois moderne,la phonologie historique est indispensable.

Certes la diachronie peut déterminer l'origine d'une forme actuelle, tout comme le latin donne l'étymon et permet de reconstituer les changements historiques d'un mot, mais le français moderne fonctionne indépendamment de la diachronie. Depuis Hartman et Hockett, la combinaison des consonnes et voyelles du mandarin a déjà fait objet de plusieurs études synchroniques.

Plus loin, l'auteur assimile la prononciation à l'écriture (Tang 2002, p. 15) :

Étudier yīnyùnxué 音韻學, c'est d'abord analyser la prononciation des sinogrammes et connaître les particularités de la structure phonologique du chinois. Nous savons que les langues indo-européennes qui utilisent l'alphabet ont comme unité d'analyse les phonèmes quand on étudie leur phonétique. Mais nos sinogrammes ne sont pas alphabétiques, (...) l'unité d'analyse de la phonétique des sinogrammes est la syllabe. (...) Un sinogramme est égal à une syllabe et vice versa. C'est pourquoi le sinogramme est non seulement une unité de l'écriture, mais aussi une unité de la phonétique. 
Ensuite, l'auteur, parlant de la prononciation rapide de deux syllabes, mêle phonologie et phonétique

[Un sinogrammes est égal à une syllabe] c'est le cas en général. Il y a aussi des cas particuliers : lorsque les gens parlent très vite, deux sinogrammes sont prononcés dans une seule syllabe,...

Ces exemples suggèrent que cette discipline est l'héritière d'une tradition qui place les sinogrammes au centre de la linguistique chinoise, bien qu'elle contrevienne aux enseignements de la linguistique générale et qu'elle ignore les études sur d'autres langues.

Sur le plan méthodologique, Geng (2004) résume les méthodes de yīnyùnxué 音韻學 $\mathrm{du} \mathrm{XX}$ siècle,

1. Analyse du pied

2. Comparaison des sinogrammes employés dans la méthode fănqiè 反切. Si le même sinogramme est utilisé pour transcrire l'initiale de plusieurs sinogrammes, ceux-ci sont dits avoir la même initiale.

3. Analyse des idéo-phonogrammes

4. Analyse des phénomènes de yìwén 異文 $^{18}$

5. Statistiques (dans l'abondance de documents anciens, on trouve des contradictions que la statistique peut permettre de résoudre)

6. Utilisation des lois phonétique dans la reconstruction

7. Méthode comparative

8. Reconstruction interne. Cette méthode prend le système reconstruit du $\mathrm{CM}$ comme point de départ pour reconstruire le CA

9. Comparaison des translitérations entre le chinois et les langues anciennes en contact

Parmi ces méthodes, (1), (2), (3), (4) sont directement héritées des lettrés chinois ; (7), (8), (9) représentent les apports de la linguistique occidentale du XIX ${ }^{\mathrm{e}}$ siècle ; (6) est une combinaison de la méthode traditionnelle et de la linguistique comparée ; (5) est une nouvelle méthode, mais la statistique n'est en rien une méthode scientifique. En résumé, ces méthodes n'ont qu'un rapport lointain avec la linguistique formelle de la deuxième moitié du $\mathrm{XX}^{\mathrm{e}}$ siècle.

\subsection{Yīnyùnxué 音韵學 face à l'approche sociolinguistique du changement phonétique}

Dès la politique d'ouverture, depuis 1978, yinnyùnxué 音韻學 s'est intéressée à la phonologie « occidentale », de façon différenciée. L'approche sociolinguistique de Labov a rencontré un écho dans les travaux menés au Sociolinguistics Laboratory du Département de chinois de l'Université de Nankin, notamment ceux de $\mathrm{Xu}$ Daming. En même temps, il partage la critique du sociolinguiste Gregory Guy

18 Le même mot monosyllabique qui peut s'écrire de façons différentes. 
concernant la phonologie formelle post-générative qui aurait repris l'opposition saussurienne langue/parole, synchronie/diachronie en étudiant quasiment exclusivement la phonologie synchronique ( $c f$. Xu 2003). Cette situation n'est pas sans ressemblance avec la réception des méthodes comparative et structuraliste : l'approche sociolinguistique qui trouve son l'application dans la dialectologie et la diachronie, est considérée par Xu (2003) comme « très bien accueillie en Chine », la phonologie formelle qui s'intéresse en premier à la synchronie et aux universaux semble rencontrer quelques obstacles de réception. Nous nous intéressons spécifiquement aux phonologies post-génératives et ne rentrerons donc pas dans les détails de la réception de l'approche sociolinguistique.

\subsection{Yīnyùnxué 音韵學 face aux nouvelles phonologies}

Les destins séparés du comparatisme et du structuralisme en Chine dans la première moitié du siècle dernier expliquent en partie la situation actuelle d'ignorance mutuelle des deux phonologies. Le travail de reconstruction non seulement passionne les linguistes traditionnels, mais suscite chez eux une sorte d'orgueil. On y relève souvent une intention avérée de rendre hommage à la tradition chinoise. Par exemple, He (1995a, p. 145) pense que dèngyùnxué 等韻 學, étude articulatoire et distributionnelle du son, est l'équivalent de la phonétique et de la phonologie modernes et il cite le phonéticien Wu Zongji qui soutient que cette tradition devance de «plusieurs siècles la phonologie occidentale».

Depuis la politique d'ouverture, le renouvellement des courants en phonologie formelle a provoqué plusieurs réactions chez les phonologues de yinnyùnxué 音韻學.

La première, la plus courante, les ignore. Les travaux sur l'histoire de la linguistique chinoise ne mentionnent aucune des études menées dans le cadre des nouvelles phonologies. Ils s'en tiennent aux travaux de ChaoYuen-Ren, Li FangKuei, Luo Changpei et Karlgren sans mentionner que Chao et Li sont des témoins de la phonologie de leur temps.

La deuxième réaction est de les rejeter. Une partie des chercheurs, minoritaire certes, mais qui exerce une certaine influence dans le domaine, s'en tient à cette position.

Comme yinnyùnxué 音韻學 place les sinogrammes au centre du domaine, d'aucuns développent la trinité forme-son-sens des sinogrammes à l'extrême. Le point de vue de Xu Tongqiang est représentatif de celui de bon nombre de linguistes chinois. Professeur au Département de chinois à l'Université de Pékin, c'est un des chercheurs les plus renommés dans le domaine de la linguistique diachronique. Selon lui, le chinois est une langue unique et la linguistique des langues alphabétiques ne peut pas s’y appliquer. Il préside un projet «Collection d'études du chinois basée sur les sinogrammes (Hànyǔ zì běnwèi yánjiū cóngshū 漢語字本位研究叢書) ». Il pense que la transmission des savoirs de l'Occident 
en Chine, y compris la linguistique moderne, a interrompu la tradition linguistique chinoise et il a sur ce sujet un jugement négatif. Ses arguments principaux sont exposés dans Xu (2005). Selon lui, la linguistique générale a été développée à partir des langues indo-européennes, qui ont une morphologie riche, et toute étude en Occident s'organise autour du mot et ses fonctions syntaxiques, mais il n'y a pas de notions correspondant en chinois à la notion de mot, seulement des sinogrammes syllabiques. La notion de mot a été imposée par la linguistique occidentale.

Contre cette conception, plusieurs arguments se présentent. D'abord, il y a des langues pluri-syllabiques qui utilisent ou utilisaient les sinogrammes chinois. Le japonais est une langue altaïque, plurisyllabique, et un sinogramme peut y représenter deux syllabes voire trois ; il n'y a pas d'équation « un sinogramme $=$ une syllabe » comme en chinois. C'est un témoignage de ce que le système d'écriture chinoise est transposable, transférable à des langues typologiquement différentes et que la langue et l'écriture fonctionnent bien sur deux paliers distincts. Ensuite, beaucoup de mots dialectaux ne sont pas transcriptibles avec les sinogrammes. Un dialecte culturellement prépondérant comme le cantonais a choisi de créer des sinogrammes spécifiques pour transcrire certaines syllabes qui sont propres à ce parler. Si les homophones semblent plaider pour l'écriture en tant que « deuxième langue » des Chinois (Saussure), ce n'est pas le seul moyen de dissiper l'ambiguité.

Xu s'inscrit dans le courant nationaliste qui considère que l'étude du chinois ne doit pas prendre pour modèle la linguistique occidentale :

Nous étudions ce qu'ils ont comme concepts (...) pour tourner en rond ; à peine avons-nous compris ce que font les Occidentaux, leurs théories changent déjà, et nous nous engageons tout de suite dans leur nouvelle théorie pour continuer à tourner en rond. (Xu 2005)

Il cite Lü (1986), disant que dès lors que toutes les théories viennent de l'étranger, nous courrons toujours après la dernière. Lü, plus familier avec le structuralisme, avait du mal à comprendre à la fin de sa carrière le générativisme et le renouvellement du formalisme. La transmission des théories linguistiques n'est pas seulement liée à la compréhension de ces théories, c'est aussi la compréhension du contexte de ces théories et des causes de leur évolution. Quand Xu écrit : « nous sommes à une époque où l'émergence de notre pays et de notre nation a réveillé la conscience qu'il nous fallait faire la recherche avec notre propre créativité » et qu'il faut « rattraper et dépasser les Occidentaux » puis arrivant à sa conclusion : « Toute discipline [chinoise] doit avoir sa propre théorie, la linguistique [chinoise] aussi, sinon, nous ne serons que des vassaux des théories occidentales », il transfère en linguistique une conception politique.

Le « miracle » chinois en économie a provoqué l'émergence d'un courant nationaliste en sciences humaines qui se revendique des valeurs traditionnelles, en 
particulier les guóxué 國學, les « savoirs chinois». S'il est admis que les sciences dures n'ont pas de patrie, en sciences humaines, le problème n'est pas aussi simple à trancher. Développer des théories chinoises pour concurrencer les Occidentaux en sciences humaines fait partie aujourd'hui d'une politique implicite de l'État. Le propos de Xu (2005) n'est qu'un des témoignages d'un courant global : « retourner à nos traditions ».

Une troisième attitude, moins fréquente, revient à adopter les nouvelles phonologies. Il y a très peu de chercheurs formés dans la tradition chinoise ouverts aux nouvelles phonologies. Feng Shengli et Wang Hongjun en sont les seuls exemples à notre connaissance. Feng Shengli a été formé en linguistique traditionnelle chinoise puis il est parti aux États-Unis dans les années 80 pour étudier la syntaxe historique et la théorie métrique de Liberman. Plus syntaticien que phonologue, il a appliqué la théorique métrique à la syntaxe historique, y compris celle des textes rimés chinois. Wang Hongjun, élève de Xu Tongqiang, après une formation en yīnyùnxué 音韻學, s'est formée en phonologie au MIT en tant que chercheur invité. Elle défend la nécessité du renouvellement de la méthodologie :

il semble depuis des années que les problèmes théoriques [du chinois] ont été résolus une fois pour toutes, il ne resterait aux chercheurs qu'à décrire avec la même méthodologie la phonologie de tous les dialectes chinois. Ceci paraît illusoire. (Wang 1999, p. 36)

Le point de vue de Wang sur la structure syllabique du chinois s'oppose évidemment à celui de son maitre, Xu (2005). Yìnyùnxué 音韻學 qui focalise très tôt sur la structure syllabique prend un caractère phonémique, mais faute de comparaison avec d'autres langues pour cause d'ethnocentrisme, la structure syllabique a été considérée comme une particularité du chinois :

Yinnyùnxué 音韻學 traditionnelle a proposé de diviser l'attaque et la rime, mais elle n'a pas considéré que c'est un des universaux des langues humaines, au contraire, [cette structure] a été considérée comme valable uniquement pour le chinois (...) À cause de l'ignorance des autres langues et du manque de conscience de l'universel dans le particulier, la linguistique chinoise a manqué l'occasion de découvrir le modèle syllabique au sens de la linguistique générale. (Wang 1999, p. 12)

\section{CONCLUSION}

Dans cet article nous avons, après un rappel de la tradition chinoise en phonologie, dessiné le paysage phonologique du chinois $\mathrm{du} \mathrm{XX}^{\mathrm{e}}$ siècle, pour essayer de comprendre la divorce de la diachronie et de la synchronie dans les études du chinois. La transmission des théories linguistiques occidentales en Chine n'est pas neutre. Le comparatisme, centré sur la diachronie et qui vise à élargir son champ 
de comparaison, a trouvé en Chine des partisans enthousiastes et a participé ainsi au travail de reconstruction du CM et CA, l'objectif des lettrés chinois depuis des générations. Le structuralisme, qui assurait un futur à la linguistique en considérant l'état synchronique d'une langue comme un système, n'a eu que peu d'écho. La séparation de deux phonologies, la phonologie chinoise, qui étudie le chinois comme une langue particulière, et la phonologie du chinois, qui étudie le chinois dans le cadre de la linguistique générale, était déjà actée à cette époque.

$\mathrm{Si}$, avant 1949, les linguistes chinois étaient informés du développement théorique du structuralisme, même si celui-ci n'était pas partout reçu, après cette date et pendant trente ans, la linguistique chinoise s'est trouvée complètement isolée. Après la réouverture du pays, les linguistes chinois ne pouvaient plus suivre le renouvellement du formalisme en linguistique. Ils éprouvaient peu d'intérêt pour les courants contemporains en phonologie et continuaient à travailler dans un cadre comparatiste. Dans ce contexte, la séparation des deux phonologies est devenue définitive.

La phonologie chinoise n'est pas la seule qui ait ses propres traditions méthodologique et terminologique. Les romanistes, germanistes et slavisants travaillent aussi d'une façon idiosyncrasique en diachronie ${ }^{19}$. Pourtant depuis une dizaine d'années, des travaux sur la diachronie des langues romanes, germaniques et slaves dans le cadre des nouvelles phonologies s'avèrent très stimulants, dans le sens où les modèles phonologiques expliquent pourquoi un phénomène en diachronie s'est produit de telle façon et non pas autrement ( $c f$. par exemple les travaux de Carvalho (2008 ab) et de Ségéral \& Scheer $(2001,2008)$ sur la diachronie des langues romanes). Un renouvellement du cadre théorique (le comparatisme) a déjà permis à la phonologie chinoise de progresser dans les années 20-40 du siècle dernier, une attitude ouverte aux nouvelles phonologies ne pourrait que lui être bénéfique.

\section{BIBLIOGRAPHIE}

Bao, Zhiming, 1990. On the nature of tone, thèse de doctorat, MIT.

- 1999. The structure of tone, New York, Oxford University Press.

Chao, Yuen-Ren, 1929. "Beiping yudiao de yanjiu [Études de l'intonation du pékinois] », dans Zuihou wu fenzhong [Les dernières cinq minutes], Annexe, Pékin, Presse Commerciale.

- 1968. A grammar of spoken Chinese, Berkeley/Los Angeles, University of California Press.

19 À titre d'exemple, Caratini (2009, chap. 2) confronte deux approches linguistiques de l'allemand, générative et néogrammairienne, une conception qui n'est pas sans ressemblance avec la différence des deux méthodes phonologiques sur le chinois exposées dans ce chapitre. L'approche néogrammairienne remonte à une longue tradition de la philologie comparée allemande, l'approche générative est apparue un siècle plus tard. La tradition philologique est centrée sur la diachronie, la grammaire générative rend compte des phénomènes en synchronie. 
- 2002. Zhao Yuanren yuyanxue lunwen ji [Travaux linguistiques de Chao Yuen-Ren], Pékin, Presse commerciale.

Caratini, Émilie, 2009. Vocalic and consonantal quantity in German: synchronic and diachronic perspectives, thèse de doctorat, Université de Nice.

Carvalho, Joaquim Brandão de, 2008a. "Western Romance », dans Joaquim Brandão de Carvalho, Tobias Scheer \& Philippe Ségéral (éds.), Lenition and fortition, Berlin, Mouton-DeGruyter, 207-233.

- 2008b, "From positions to transitions: A contour-based account of lenition », dans Joaquim Brandão de Carvalho, Tobias Scheer \& Philippe Ségéral (éds.), Lenition and fortition, Berlin, Mouton-DeGruyter, 415-445.

Chen, Matthew Y., 2000. Tone sandhi: Patterns across Chinese dialects, Cambridge University Press.

Cheng, Chin-Chuan, 1973. A synchronic phonology of Mandarin Chinese, The Hague, Mouton.

Dell, François, 1973. « Two cases of exceptional rule ordering », dans Ferenc Kiefer \& Nicolas Ruwet (eds.), Generative grammar in Europe, Dordrecht, Reidel, 141-153.

- 1994. "Consonnes à prolongement syllabique en Chine ", Cahiers de linguistique Asie orientale, 23 (1), 87-94.

- 2004. " On recent claims about stress and tone in Beijing Mandarin », Cahiers de linguistique - Asie orientale, 33 (1), 33-63.

Duanmu, San, 1990. A formal study of syllable, tone, stress and domain in Chinese languages, thèse de doctorat, MIT.

- 2007. The phonology of standard Chinese, NewYork, Oxford University Press [2000].

Feng, Shengli, 1997. Hanyu de yunlv, cifa yu jufa [La métrique, la morphologie et la syntaxe du chinois], Presse Universitaire de Pékin.

Ferlus, Michel, 2009. « What were the four divisions of Middle Chinese? », Diachronica, 26 (2), 184-213.

Geng, Zhensheng, 2004. Ershi shiji hanyu yinyunxue fangfalun [Méthodologie de la phonologie chinoise du $\mathrm{XX}^{\mathrm{e}}$ siècle], Presse Universitaire de Pékin.

Goh, Yeng-Seng, 1997. The segmental phonology of Beijing Mandarin, Taipei, The Crane Publishing Co.

Gong Hwang-cherng, 2004. Hanzangyu yanjiu lunwen ji [Recueil des études sinotibétaines], Pékin, Presse universitaire de Pékin.

Hartman, Lawton M., 1944. « The segmental phonemes of the Peiping dialect », Language, 1944, 28-42.

He, Jiuying, 1995a. Zhongguo gudai yuyanxue shi [Histoire de la linguistique en Chine ancienne], Canton, Éditions pédagogiques du Guangdong.

- 1995b. Zhongguo xiandai yuyanxue shi [Histoire de la linguistique en Chine moderne], Canton, Éditions pédagogiques du Guangdong.

Hockett, Charles Francis, 1947. «Peiping phonology », Journal of the American Oriental Society, 67 (4), 253-267.

- 1950. « Peiping morphophonemics », Language, 26 (1), 63-85.

$\mathrm{Hu}$, Anshun, 2003. Yinyunxue tonglun [Introduction générale de la phonologie chinoise], Pékin, Librairie de Zhonghua.

Jones, Daniel \& Woo, Kwing Tong, 1912. A Cantonese phonetic reader, University of London Press.

Kaye, Jonathan, 2001a. « A fresh look at putonghua onset-rime pairs », Ms.,Guangdong University of Foreign Studies.

- 2001b. «A short theory about tones », Ms., Guangdong University of Foreign Studies.

Liu, Lili, 2004. «Ershi shiji hanyu shengdiao lilun de yanjiu zongshu [Études des theories sur les tons chinois au $\mathrm{XX}^{\mathrm{e}}$ siècle] », Dangdai Yuyanxue [Linguistique contemporaine], 2004 (1), 45-56.

Liu, Te-hsin, 2008. Marque, registre et contour dans les systèmes tonals en chinois, thèse de doctorat, Université Paris VIII. 
Luo, Changpei, 1956. Xiamen yinxi [Phonologie du xiamen], Pekin, Éditions des sciences.

Luo, Xiaoliang, 2013. Vers une phonologie CVCV du chinois : analyse des interactions entre syllabe, ton et processus morphologiques, thèse de doctorat, Université d'Orléans.

Lü, Shuxiang, 1986. "Zhongguo yufaxue shigao xu [Préface de l'histoire de la grammaire chinoise] », dans Zhongguo yufaxue shigao [Histoire de la grammaire chinoise], 1-2, Pékin, Éditions Langage et Culture.

Martin, François, 1988. « Système et prosodie : questions relatives aux tons et au contrepoint tonal dans la tradition chinoise », Extrême-Orient, Extrême-Occident, 10, 95-107.

- 1990. « Note sur l'histoire de la série des quatre tons ", Extrême-Orient, ExtrêmeOccident, 12, 67-87.

Malmqvist, Göran, 2011. Bernhard Karlgren: Portrait of a Scholar, Bethlehem, Lehigh University Press.

Sagart, Laurent, 1999. The root of Old Chinese, Amsterdam, John Benjamins Publishing Campany.

Ségéral, Philippe \& Scheer, Tobias, 2001. « La coda mirior », Bulletin de la Société de Linguistique de Paris, 96 (1), 107-152.

- 2008. «Positional factors in lenition and fortition », dans Joaquim Brandão de Carvalho, Tobias Scheer \& Philippe Ségéral éds., Lenition and fortition, Berlin : MoutonDeGruyter, 131-172.

Sun, Yizhi, 2006. Anhui jianghuai guanhua yuyin yanjiu [Études de la phonétique des mandarins de l'Anhui], Hefei, Librairie de la Montagne Jaune.

Tang, Zuofan, 2002. Yin yun xue jiaocheng [Cours de phonologie chinoise], Presse Universitaire de Pékin.

Tang, Zuofan \& Geng, Zhensheng, 1998. « Ershi shiji de hanyu yin yunxue [La phonologie chinoise du vingtième siècle] », dans Jian Liu (ed.), Ershi shiji de zhongguo yuyanxue [La linguistique chinoise du vingtième siècle], Presse Universitaire de Pékin.

Wang, Hongjun, 1999. Hanyu Feixianxing Yinxixue [Phonologie non linéaire du chinois], Presse Universitaire de Pékin.

Wang, Li, 1980. Hanyu shigao [Histoire du chinois], Librairie de Zhonghua [1957].

- 1981. Zhongguo yuyanxue shi [Histoire de la linguistique chinoise], Taiyuan, Editions du Peuple du Shanxi.

Woo, Nancy, 1969. Prosody and Phonology, thèse de doctorat, MIT.

$\mathrm{Xu}$, Daming, 2003. "Yuyan yanjiu de kexuehua [La scientification de l'étude linguistique] ", Yuyan jiaoxue yu yanjiu [Enseignementet recherche des langues] 2003 (1), 17-28.

$\mathrm{Xu}$, Tongqiang, 2005. " $\mathrm{Zi}$ benwei he yuyan yanjiu [ $\mathrm{Zi}$ en tant que structure de base et recherches linguistiques] ", Yиyan jiaохие уи уапjiu [Enseignementet recherche des langues], 2005 (6), 1-11.

Yip, Moira, 1980. The tonal phonology of Chinese, thèse de doctorat, MIT.

- 1995. " Tone in east asian languages », dans John Goldsmith (ed.), The handbook of phonological theory, Cambridge, MA, Blackwell, 476-494.

- 2002. Tone, New York, Cambridge University Press.

Zhao, Zhenduo, 2000. Zhongguo yuyanxue shi [Histoire de la linguistique chinoise], Shijiazhuang, Éditions pédagogiques du Hebei.

Zhu, Jianing, 2013. "Suoxuer lishi yuyan guan zai shengyunxue shang de yingyong » [L'application de la conception diachronique de Saussure dans l'étude de la phonologie chinoise], communication présentée au colloque international « La linguistique structurale à l'épreuve de sa réception : l'exemple de Saussure », Nankin, Chine. 